\title{
The Effect of Tartaric and Citric Acid as a Complexing Agent on Defect Structure and Conductivity of Copper Samarium Co-doped Ceria Prepared by a Sol-Gel Auto-Combustion Method
}

Kornrawit Duangsa

Khon Kaen University

Apishok Tangtrakarn ( $\square$ nateta@kku.ac.th )

Khon Kaen University https://orcid.org/0000-0002-8351-5215

Charustporn Mongkolkachit

National Metal and Materials Technology Center (MTEC)

Pavadee Aungkavattana

National Nanotechnology Center (NANOTEC)

Klitsada Moolsarn

Khon Kaen University

Keywords:

Posted Date: January 18th, 2021

DOI: https://doi.org/10.21203/rs.3.rs-146185/v1

License: (c) (i) This work is licensed under a Creative Commons Attribution 4.0 International License.

Read Full License 


\section{Abstract}

The authors have requested that this preprint be removed from Research Square. 